\title{
Paramagnetically Shifted High-Resolution Proton NMR Spectra of Poly(methyl methacrylate) by the use of Tris(dipivalomethanato)europium(III).
}

\author{
Shigetoshi Amiya, Isao Ando, and Riichirô ChûJô \\ Department of Polymer Engineering, Tokyo Institute of Technology, \\ 12-1, 2-Chome, Ookayama, Meguro-ku, Tokyo 152, Japan.
}

(Received June 29, 1972)

\begin{abstract}
Tris(dipivalomethanato)europium(III), as the NMR shift reagent has been applied to the NMR measurement of four types of poly(methyl methacrylate) samples in benzene solution. The time dependences of the chemical shifts of the polymers were measured. Chemical shifts become larger and larger till about $100 \mathrm{~min}$ after the addition of the shift reagent in polymer solutions and subsequently they did not vary further for several hours. It is found that the isotropic shifts of these polymers are inversely proportional to the absolute temperature between 300 and $373^{\circ} \mathrm{K}$, all the polymer signals shift to lower fields linearly with increasing amounts of the shift reagent. Moreover the separation of the methoxy signal attributable to the pentad tacticity was observed.

KEY WORDS Poly(methyl methacrylate) / NMR / Shift Reagent /

Time Dependence / Stereospecificity / Pentad / Stereoblock /
\end{abstract}

Recently many studies have reported the use of tris(dipivalomethanato)europium(III), (hereafter it is abbreviated as $\left.\operatorname{Eu}(\mathrm{DPM})_{3}\right)$, as a shift reagent in NMR spectra analysis of low molecular weight organic compounds. ${ }^{1-4}$ There are some investigations relating to the application of the $\operatorname{Eu}(\mathrm{DPM})_{3}$ shift reagent to the NMR spectra of polymer molecules, namely the reports concerned with the application to the determination of the molecular weight of poly(propylene glycol) by NMR, ${ }^{5}$ and to the NMR spectra of atactic poly(methyl methacrylate) ${ }^{6}$ (PMMA). The authors have previously analyzed NMR spectra of copolyesters(ethylene terephthalateisophthalate) by the use of $\operatorname{Eu}(\mathrm{DPM})_{3},{ }^{7}$ as a result, the shift reagent $\operatorname{Eu}(\mathrm{DPM})_{3}$ is very effective in analyzing NMR spectra of polymers. This paper deals with the influence of the $\mathrm{Eu}(\mathrm{DPM})_{3}$ shift reagent on the NMR spectra of isotactic, syndiotactic, atactic and stereoblock PMMA's with respect to the time and temperature dependences and so on. The microtacticities of atactic and stereoblock PMMA's are also analyzed.

\section{EXPERIMENTAL}

Sample 1 (isotactic PMMA) was prepared with isobutylmagnesium bromide catalyst at $-60^{\circ} \mathrm{C}$ in toluene.

Sample 2 (syndiotactic PMMA) was supplied by Dr. Kotake of the Mitsubishi Rayon Co., Ltd.

Table I. Microtacticities of the samples used

\begin{tabular}{|c|c|c|c|c|c|}
\hline \multirow{2}{*}{$\begin{array}{c}\text { Sample } \\
\text { no. }\end{array}$} & \multicolumn{3}{|c|}{ Triads $^{a}$} & \multicolumn{2}{|c|}{$\begin{array}{l}\text { Number-averaged } \\
\text { sequence lengths }\end{array}$} \\
\hline & $I$ & $H$ & $S$ & $\langle i\rangle_{n}$ & $\langle s\rangle_{n}$ \\
\hline 1 & 99 & 0.5 & 0.5 & - & - \\
\hline 2 & 1.2 & 12.4 & 86.4 & 1.20 & 15.0 \\
\hline 3 & 4.6 & 43.3 & 52.1 & 1.36 & $3.82^{\mathrm{c}}$ \\
\hline 4 & 30.0 & 30.8 & 39.2 & 2.94 & 3.50 \\
\hline
\end{tabular}

a The microtacticity was determined by $60-\mathrm{MHz}$ NMR spectrometer.

b The number-averaged sequence lengths are obtained by the relations ${ }^{8}$

$$
\begin{array}{ll}
\langle i\rangle_{n}=1 / P_{s i} & \langle s\rangle_{n}=1 / P_{i s} \\
\langle i\rangle_{n}=1 / P_{s} & \langle s\rangle_{n}=1 / P_{i} .
\end{array}
$$

c Since the radical stereospecific polymerization is known to follow the Bernoulli mechanism, eq 2 was used to obtain the number-averaged sequence lengths, where the designation $\boldsymbol{P}_{s i}$ means the probability occurring for an isotactic dyad to a syndiotactic chain end, etc., and $\boldsymbol{P}_{i}$ and $\boldsymbol{P}_{\boldsymbol{s}}$ mean the probability occurring for an isotactic and a syndiotactic dyads, respectively, irrespective of the preceding units. 
Sample 3 (atactic PMMA) was prepared with $\alpha, \alpha^{\prime}$-azobisisobutylonitorile catalyst at $100^{\circ} \mathrm{C}$ in toluene.

Sample 4 (stereoblock PMMA) was prepared with di- $n$-butylmagnesium catalyst at $0^{\circ} \mathrm{C}$ in toluene.

A fixed quantity of the shift reagent was introduced into a polymer solution in a sample tube, and this sample tube was heated till the shift reagent dissolved. Subsequently after about ten minutes, the NMR spectra were measured in benzene solutions $(0.05 \mathrm{~g} / \mathrm{m} l)$ with a JNM-PS$100 \mathrm{NMR}$ spectrometer $(100 \mathrm{MHz})$. The temperature of the sample was kept at constant of $80^{\circ} \mathrm{C}$ unless explicitly indicated.

The microtacticities of the samples used were determined from their proton NMR spectra without the shift reagent. These values are tabulated in Table I.

\section{RESULTS AND DISCUSSION}

The Influence of the $\mathrm{Eu}(\mathrm{DPM})_{3}$ Shift Reagent on

\section{Polymer}

The time dependences of the chemical shifts were observed for the polymer in solution after the addition of the $\operatorname{Eu}(\mathrm{DPM})_{3}$. Figure 1 shows that the chemical shifts of the polymers become larger and larger during $100 \mathrm{~min}$ after the addi- tion of $\mathrm{Eu}(\mathrm{DPM})_{3}$ to polymer solutions. (Where the actual time of adding the shift reagent into a sample tube is defined as a reference of time $(t=0)$ : In practice the point plotted for $t=0$ corresponds to the data without addition of shift reagent.) Subsequently during the next few hours the chemical shifts did not vary further, while after more than $24 \mathrm{hr}$ the magnitude of the shift (from the original chemical shift without the addition of $\operatorname{Eu}(\mathrm{DPM})_{3}$ ) decreased. After more than $24 \mathrm{hr}$ a semitransparent substance formation was recognized at the bottom of the sample tube. The cause for the production of this substance is not clear as yet. The relative chemical shift remains constant after the production of this substance. Such a time dependence cannot be caused by the exchange between polymers in an associated state and in a nonassociated state with $\mathrm{Eu}(\mathrm{DPM})_{3}$, but by the diffusion of $\mathrm{Eu}(\mathrm{DPM})_{3}$ in the polymer solution. If the former is the case, the NMR spectra due to the complex of $\operatorname{Eu}(\mathrm{DPM})_{3}$-substrate and due to the polymer free from complex formation might be observed separately, because such wide range of time dependence requires rather small rate of exchange. The "diffusion" means a process for reaching an equilibrium state in the concentration of the shift reagent. This process may required a rather long period because of the

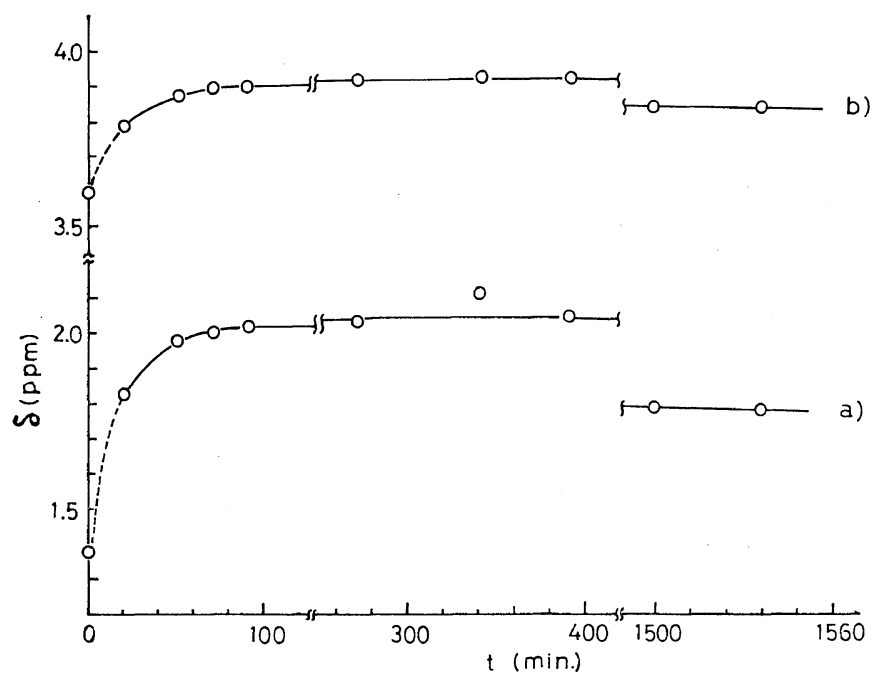

Figure 1. The time dependence of the chemical shifts after the addition of the $\mathrm{Eu}(\mathrm{DPM})_{3}\left(8.6 \times 10^{-2} \mathrm{~mol}\right.$ to PMMA) for the sample 1: (a) $\alpha-\mathrm{CH}_{3}$; (b) $\mathrm{O}-\mathrm{CH}_{3}$ groups. 
concentration gradient of the solute macromolecules in solution even in a dilute solution.

The temperature dependence of the NMR spectra is also an interesting problem. However, it is impossible or very difficult to closely follow this type of dependence in samples without the semitransparent substance formation. Instead, we used the samples for which more than $24 \mathrm{hr}$ had elapsed. This may be permitted because of the constancy of the shift in this time region.

It is well known that isotropic proton reasonance shifts caused by the compounds including the transition and the rare earth elements may arise from two sources, contact and/or pseudocontact interactions.

The contact interaction shift is described by eq $1^{9}$

$$
\left(\frac{\Delta H}{H}\right)_{i}=-A_{\mathrm{i}} \frac{\gamma_{\mathrm{e}}}{\gamma_{\mathrm{H}}} \frac{g \beta S(S+1)}{3 k T}
$$

where $A_{\mathrm{i}}$ is the isotropic hyperfine contact interaction constant for the $i$-th proton and where the other symbols have their usual meanings. ${ }^{10}$

For ions with $\mathrm{C}_{2 v}$ or $\mathrm{C}_{2}$ symmetry the pseudocontact shift experienced by the $i$-th proton in a complex is given by ${ }^{11}$

$$
\begin{aligned}
\left(\frac{\Delta H}{H}\right)_{i}= & -\varepsilon\left(g_{1}-g_{2}-g_{3}\right) \\
& \times\left[\frac{\left(g_{1}-\frac{1}{2} g_{2}-\frac{1}{2} g_{3}\right)\left(3 \cos ^{2} \chi_{i}-1\right)}{R_{i}{ }^{3}}\right. \\
& \left.-\frac{\frac{3}{2}\left(g_{2}-g_{3}\right) \sin ^{2} \chi_{i} \cos 2 \Omega}{{R_{i}}^{3}}\right]
\end{aligned}
$$

where $\varepsilon=|\beta|^{2} \cdot S(S+1) / 2 k T$, the distance between the metal and the $i$-th proton, $R_{i}$, and the angles $\chi_{i}$ and $\Omega_{i}$ define its position of the proton within the frame of the symmetry coordinates of the metal ion. The quantities $g_{1}, g_{2}$, and $g_{3}$ are the diagonal components in $g$ tensor. Namely, for the shifts produced by both contact and pseudocontact interactions, the general expectation is that the shift produced will be inversely proportional to temperature. Figure 2 supports theoretically the above mentioned phenomenon.

Figures 3-6 show the change of the chemical shifts of the three groups $\left(\alpha-\mathrm{CH}_{3}, \mathrm{CH}_{2}\right.$, and $\mathrm{O}$ $\mathrm{CH}_{3}$ ) of samples $1-4$ with increasing concentration of $\mathrm{Eu}(\mathrm{DPM})_{3}$. In the case of sample 1 as shown in Figure 3, the order of the magnitude of the shift of each group due to the shift reagent is as follows; $\mathrm{CH}_{2}>\alpha-\mathrm{CH}_{3}>\mathrm{OCH}_{3}$, and in the case of sample 2, $\alpha-\mathrm{CH}_{3}>\mathrm{CH}_{2}>\mathrm{OCH}_{3}$. The methoxy peak is hardly affected in either case, the change in the chemical shifts of the methylene protons of sample 1 is larger than that of sample 2 , and for the $\alpha$-methyl protons, sample 2 is more

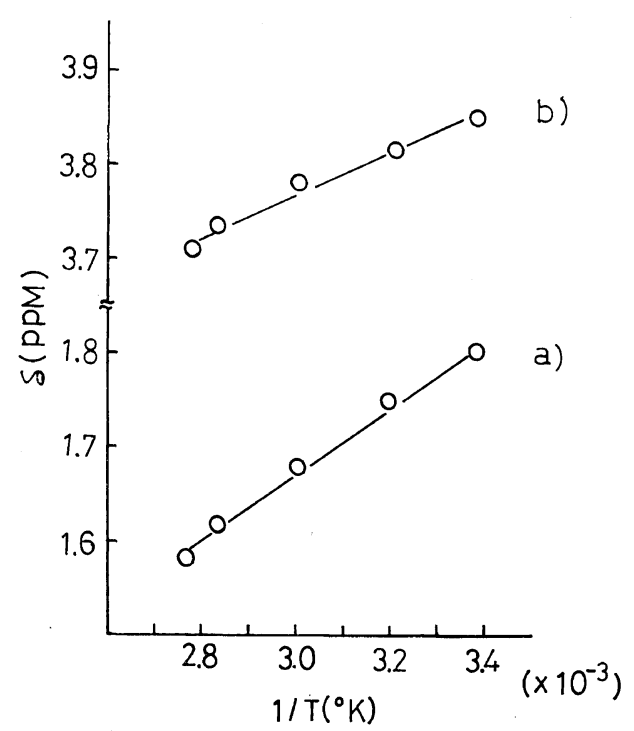

Figure 2. Plots of the chemical shifts for $1 / T$ : (a) $\alpha-\mathrm{CH}_{3}$; (b) $\mathrm{O}-\mathrm{CH}_{3}$ groups. Measurements are with taken after $24 \mathrm{hr}$ or more from the addition of the $\operatorname{Eu}(\mathrm{DPM})_{3}$ to sample 1 .

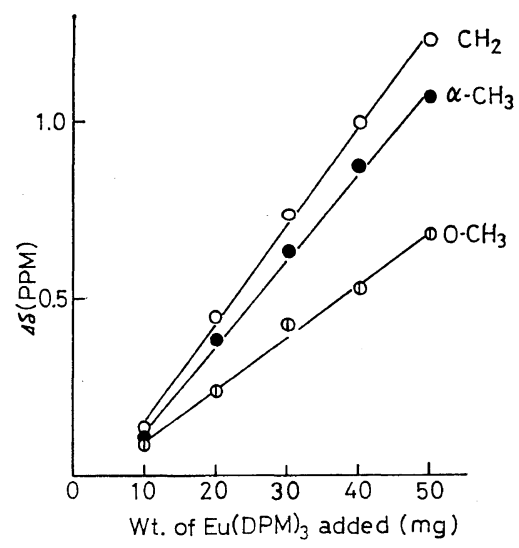

Figure 3. Variation in the chemical shift for the different protons of sample 1 solution $(0.05 \mathrm{~g} / \mathrm{ml})$ in benzene with increasing concentration of Eu$(\mathrm{DPM})_{3}$. 


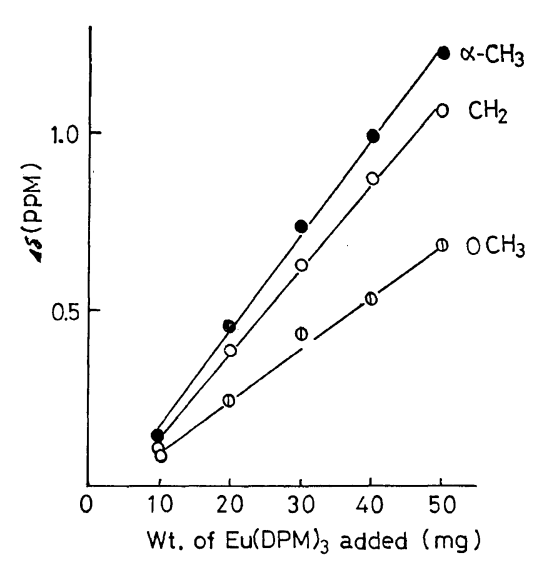

Figure 4. Variation in the chemical shift for the different protons of sample 2 solution $(0.05 \mathrm{~g} / \mathrm{m} l)$ in benzene with increasing concentration of Eu$(\mathrm{DPM})_{3}$.

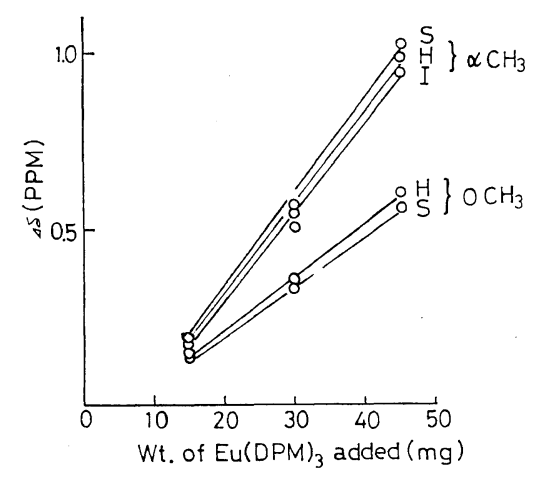

Figure 5. Variation in the chemical shift for the different protons of sample 3 solution $(0.05 \mathrm{~g} / \mathrm{m} l)$ in benzene with increasing concentration of Eu$(\mathrm{DPM})_{3}$.

affected than that of sample 1 . It is considered that these results may arise by the different configuration and conformations of the respective polymers. Especially with respect to the difference of the conformation, the geometric structure is clearly recognized as follows; isot. PMMA takes $5 / 1^{12}$ or $5 / 2$ helix conformation and synd. PMMA probably takes more extended conformation than isot. PMMA.

When the paramagnetic center is a rare earth ion, the pseudocontact shift as shown in eq 2 is probably dominant. ${ }^{13}$ Therefore usual informations concerning the geometrical structure of $\mathrm{Eu}(\mathrm{DPM})_{3}$ - PMMA system may be obtained by a plot of $\Delta \delta$ against values of $\left(3 \cos ^{2} \chi_{i}-1\right) / R_{i}{ }^{3}$

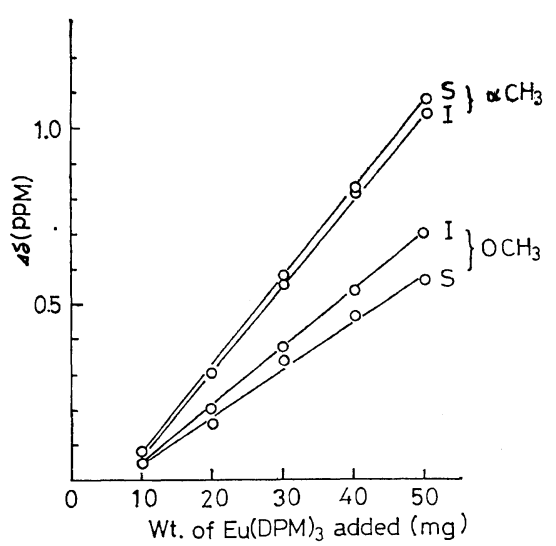

Figure 6. Variation in the chemical shift for the different protons of sample 4 solution $(0.05 \mathrm{~g} / \mathrm{ml})$ in benzene with increasing concentration of Eu$(\mathrm{DPM})_{3}$.

and/or $\sin ^{2} \chi_{i} \cos ^{2} \Omega / R_{i}{ }^{3}$ (in eq 2) for both conformations.

The shift $(\Delta \delta)$ for our systems decreases with $1 / R_{i}{ }^{3}$ which contradicts theoretical predictions. We must take into account of the angular factors $\left(\chi_{i}, \Omega_{i}\right)^{11}$ as well as the distance $R_{i}$.

This is especially important for the polymer molecule because of the wide variety of conformations.

For samples 3 and 4, when further shift reagent was added, three initially distinguishable peaks (isotactic $(I)$, heterotactic $(H)$ and syndiotactic $(S)$ triads) in the $\alpha-\mathrm{CH}_{3}$ proton region have coalesced by a decrease in the difference of these chemical shifts. The dissolution of $\mathrm{Eu}(\mathrm{DPM})_{3}$ decreases with increasing concentration of polymer in the solution, so that the addition of $\operatorname{Eu}(\mathrm{DPM})_{3}$ in excess precipitates yellow crystals even if the temperature of the samples is elevated. This is a reason why the peaks of the NMR spectra are broadened. In contrast, the peaks attributable to $I, H$, and $S$ triads of the methoxy protons are further separated by subsequent addition of $\mathrm{Eu}(\mathrm{DPM})_{3}$. As a result of the broadening, we could not assign signals in the methylene region. We did compare the chemical shift of the respective $I$ triad peak of the $\alpha-\mathrm{CH}_{3}$ protons of samples 1,3 , and 4 with each other and discussed an effect of the shift reagent on NMR spectra as a function of the number-averaged sequence lengths of the sample. This result is 
NMR of PMMA Studied by the use of $\mathrm{Eu}(\mathrm{DPM})_{3}$

reproduced in Figure 7. For samples 2-4, we now followed the same procedure for each $S$ triad peak of the $\alpha-\mathrm{CH}_{3}$ and $\mathrm{O}-\mathrm{CH}_{3}$ protons as shown in Figure 8. A comparison of the results in Figures 7 and 8 with the data of $\langle i\rangle_{n}$, and $\langle s\rangle_{n}$ shown in Table I indicates that the coordination of the shift reagent to the polymer may be easy if the sequences of the same configuration are considerably long. On the contrary, when the sequence is so short, the coordination may be difficult under the influence of the neighboring different sequence. As for the methoxy protons, if further $\mathrm{Eu}(\mathrm{DPM})_{3}$ was added, we could not assign the peaks especially of the $I$ triad corresponding to the $n$-ad microtacticity

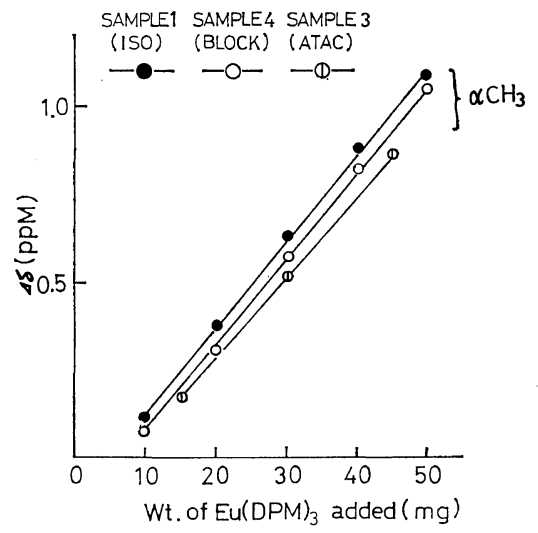

Figure 7. The comparison of the chemical shift of isotactic triad peak of $\alpha-\mathrm{CH}_{3}$ protons and that of $\mathrm{O}-\mathrm{CH}_{3}$ protons of the samples 1,3 , and 4 with increasing concentration of $\mathrm{Eu}(\mathrm{DPM})_{3}$.

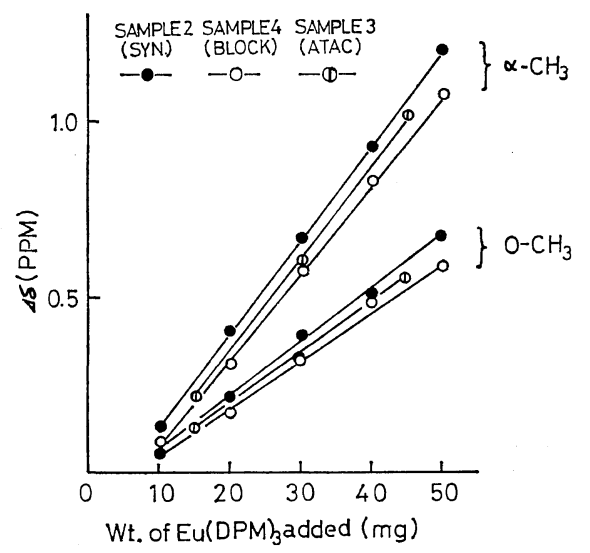

Figure 8. The comparison of the chemical shift of isotactic triad peak of $\alpha-\mathrm{CH}_{3}$ protons and that of $\mathrm{O}-\mathrm{CH}_{3}$ protons of the samples 2-4 with increasing concentration of $\mathrm{Eu}(\mathrm{DPM})_{3}$.

because of the coalescence of the peaks due to further separation in this region. We take now under consideration the reason why the straight line does not pass through the origin as shown in Figures 3-6.

Assignment of the Methoxyl Signal Attributable to Pentad Microtacticity

The splitting of methoxy protons in a benzene solution attributable to the triad tacticity has been observed with $100-\mathrm{MHz}$ NMR spectrometer. As shown in Figure 9 the splitting due to pentad tacticity was observed by the addition of Eu$(\mathrm{DPM})_{3}$ into PMMA solution. However, if further $\mathrm{Eu}(\mathrm{DPM})_{3}$ is added a higher order splitting

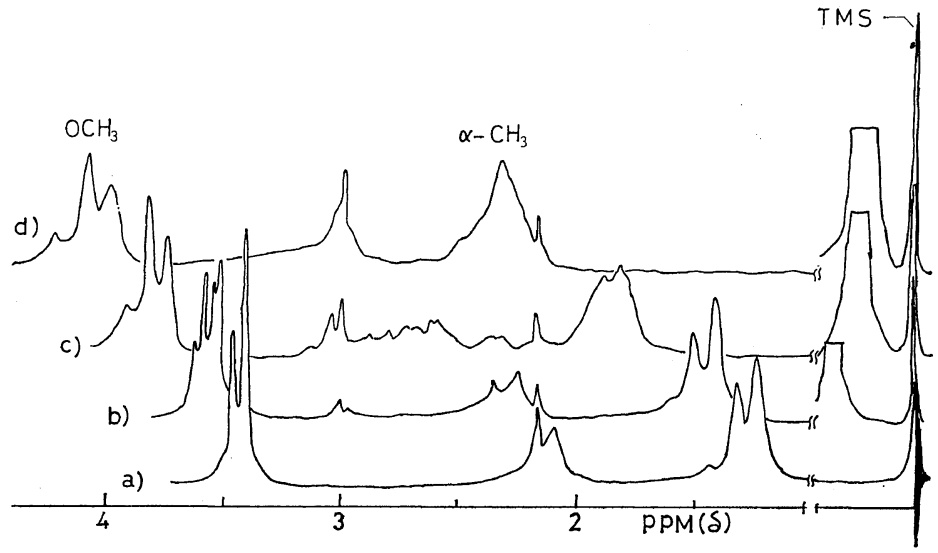

Figure 9. $100-\mathrm{MHz}$ PMR spectra of sample $3(25 \mathrm{mg})$ in benzene $(0.5 \mathrm{ml})$ containing varying amounts of $\mathrm{Eu}(\mathrm{DPM})_{3}$ : (a) $0.0 \mathrm{mg}$; (b) $15 \mathrm{mg}$; (c) $30 \mathrm{mg}$; (d) $45 \mathrm{mg}$. 
cannot be observed. In this study, when Eu$(\mathrm{DPM})_{3}\left(15 \mathrm{mg}, 8.6 \times 10^{-2} \mathrm{~mol}\right.$ to PMMA $)$ added to samples 3 and 4 in solution, the splitting due to pentad tacticity was distinctly observed.

\section{In the Case of Atactic PMMA}

Free radical polymerization is generally described by the Bernoulli trial. Thus the probability of $I, H$, and $S$ triads are calculated, using the equation ${ }^{14}$

$$
I=\sigma^{2} \quad H=2 \sigma(1-\sigma) \quad S=(1-\sigma)^{2}
$$

where $\sigma$ is the probability occurring for an isotactic dyad irrespective of the preceding units.

Table II. Pentad tacticities of the sample 3 (atactic PMMA) calculated from triad ones and obtained experimentally for the sample with $\operatorname{Eu}(\mathrm{DPM})_{3}$

\begin{tabular}{|c|c|c|c|c|c|}
\hline Triad, & $\%$ & Pentad & $\underset{\%}{\text { Calcd, }}$ & $\begin{array}{c}\text { Expt1, } \\
\%\end{array}$ & $\begin{array}{r}\text { Peak } \\
\text { no. }\end{array}$ \\
\hline \multirow{3}{*}{$S$} & \multirow{3}{*}{52.1} & rrrr & 37.0 & 39 & I \\
\hline & & rrrm & 21.0 & 20 & II \\
\hline & & mrrm & 2.9 & 27 & III \\
\hline \multirow{3}{*}{$H$} & \multirow{3}{*}{43.3} & $\mathrm{rrmr}$ & 21.0 & & \\
\hline & & $\mathrm{rrmm}+\mathrm{mrmr}$ & 11.6 & & \\
\hline & & $\mathrm{mrmm}$ & 1.7 & & \\
\hline \multirow{3}{*}{$I$} & \multirow{3}{*}{4.6} & $\mathrm{rmmr}$ & 2.9 & 14 & IV \\
\hline & & $\mathrm{rmmm}$ & 1.7 & & \\
\hline & & $\mathrm{mmmm}$ & 0.2 & & \\
\hline
\end{tabular}

\& Peak no. in Figure 10.

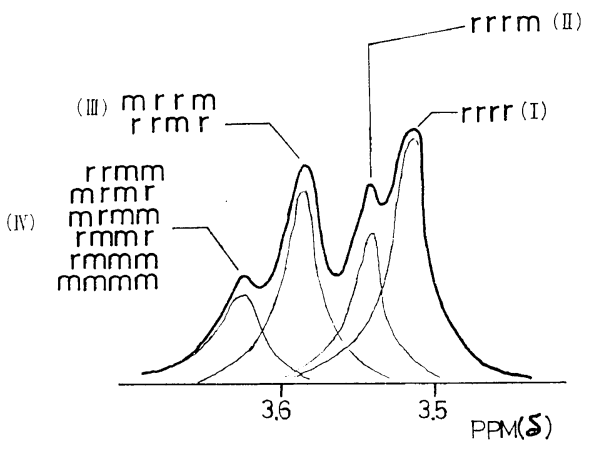

Figure 10. The assignment of pentad peaks of methoxy protons of the polymer solution in benzene in the presence of $\mathrm{Eu}(\mathrm{DPM})_{3}(15 \mathrm{mg})$ at $80^{\circ} \mathrm{C}$. (sample 3).
We have

$$
\sigma=0.22
$$

from the value listed in Table I.

Using this value we have the values for pentad probabilities as shown in Table II.

As the experimental values (the fifth column in Table II) agree well with the calculated ones (the fourth column in Table II) within the fluctuation of the experimental errors, the assignment of pentad peaks as shown in Figure 10 may be correct.

\section{In the Case of Stereoblock PMMA}

From the comparison of the triad microtacticity observed and converted from the tentatively assigned pentad microtacticity of the sample 3, each peak for the splitting of the methoxy triad signal in the sample 4 was assigned as shown in Figure 11, and the relative intensities of pentad configuration are tabulated in Table III.

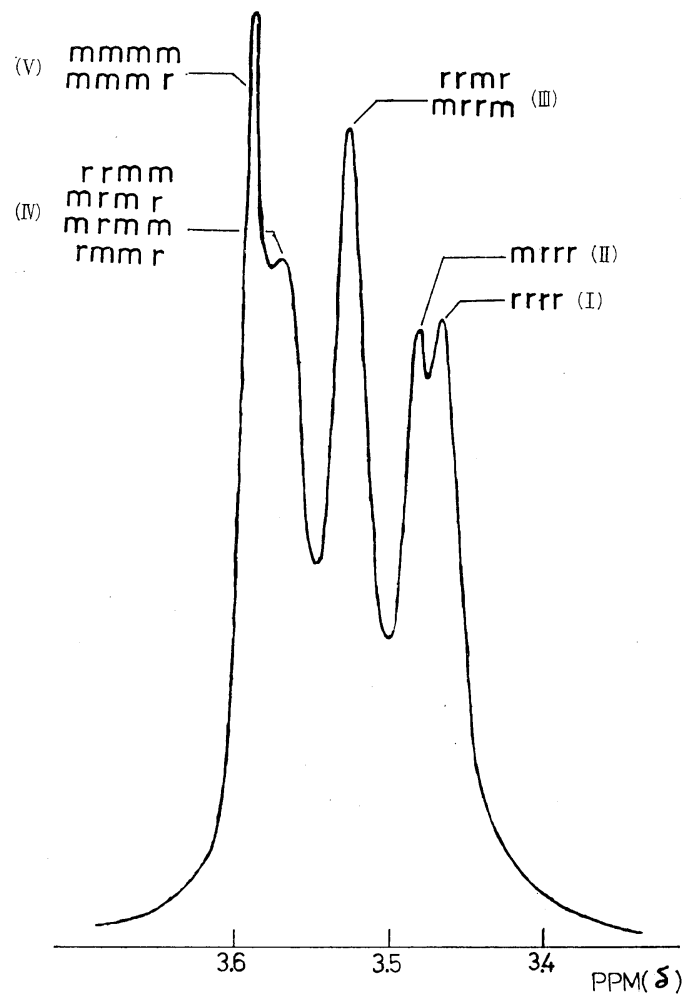

Figure 11. The assignment of pentad peaks of methoxy protons of the polymer solution in benzene in the presence of $\operatorname{Eu}(\mathrm{DPM})_{3}(15 \mathrm{mg})$ at $80^{\circ} \mathrm{C}$. (sample 4). 
Table III. Pentad tacticities of the sample 4 (stereoblock PMMA) obtained experimentally for the sample with $\mathrm{Eu}(\mathrm{DPM})_{3}$

\begin{tabular}{|c|c|c|c|c|c|}
\hline Triad, & $\%$ & Pentad & $\begin{array}{c}\text { Calcd, } \\
\%\end{array}$ & $\underset{\%}{\text { Exptl, }}$ & $\begin{array}{l}\text { Peak } \\
\text { no. }{ }^{b}\end{array}$ \\
\hline \multirow{3}{*}{$S$} & \multirow{3}{*}{38.2} & rrrr & 19 & 19 & I \\
\hline & & rrrm & 14 & 14 & II \\
\hline & & mrrm & 5 & 26 & III \\
\hline \multirow[b]{2}{*}{$H$} & \multirow[b]{2}{*}{31.8} & rmrr & 21 & 20 & 111 \\
\hline & & $\mathrm{rrmm}+\mathrm{mrmr}$ & \}$^{11}$ & 18 & IV \\
\hline \multirow[b]{2}{*}{$I$} & \multirow[b]{2}{*}{30.0} & $\mathrm{rmmr}$ & 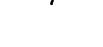 & & \\
\hline & & $\begin{array}{l}\mathrm{mmmr} \\
\mathrm{mmmm}\end{array}$ & 23 & 23 & V \\
\hline
\end{tabular}

b Peak no. in Figure 11.

\section{CONCLUSION}

The NMR spectra may be measured after at least $100 \mathrm{~min}$ and then subsequently for a few hours after the addition of $\operatorname{Eu}(\mathrm{DPM})_{3}$ to a polymer solution because the variation in the chemical shift is the largest. The lower the temperature the larger the magnitude of the shifts is, but the peaks of NMR spectra are broadened. A rather clear separation of the methoxy signal due to the pentad tacticity was observed under appropriate conditions: solvent, benzene; amount of $\mathrm{Eu}(\mathrm{DPM})_{3}, 15 \mathrm{mg}\left(8.6 \times 10^{-2} \mathrm{~mol}\right.$ to PMMA); concn of polymer solution, $0.05 \mathrm{~g} / \mathrm{ml}$; temperature, $80^{\circ} \mathrm{C}$.

\section{REFERENCES}

1. C. C. Hinckley, J. Amer. Chem. Soc., 91, 5160 (1969).

2. C. C. Hinckley, ibid., 93, 2417 (1971).

3. J. K. M. Sauders and D. H. Williams, Chem. Commun., 422 (1970).

4. J. K. M. Sauders and D. H. Williams, J. Amer. Chem. Soc., 93, 641 (1971).

5. F. F.-L.-Ho, Polymer Letter, 9, 491 (1971).

6. A. R. Kartitzky and A. Smith, Tetrahedron Lett., 21, 1765 (1971).

7. R. Chûjô, K. Koyama, I. Ando, and Y. Inoue, Polymer J., 3, 394 (1972).

8. R. Chûjô, J. Macromol. Sci., B2, 1 (1968).

9. H. M. McConnell and D. B. Chestnut., J. Chem. Phys., 28, 107 (1958).

10. D. R. Eaton and W. D. Phillips, "Advanced in Magnetic Resonance", Vol. 1, Academic Press, New York, N.Y., 1965, p 103.

11. G. N. LaMar, William DeW. Horrocks, and L. Aallen., J. Chem. Phys., 41, 2126 (1964).

12. H. Tadokoro, Y. Chatani, H. Kusanagi, and M. Yokoyama, Rep. Progr. Polym. Phys. Japan, 12, 173 (1969).

13. D. R. Eaton, J. Amer. Chem. Soc., 20, 3097 (1965).

14. F. A. Bovey and G. V. D. Tiers, J. Polym. Sci., 44, 173 (1960). 